\title{
Oral adenosine-5'-triphosphate (ATP) administration increases blood flow following exercise in animals and humans
}

\author{
Ralf Jäger ${ }^{1}$, Michael D Roberts ${ }^{2}$, Ryan P Lowery ${ }^{3}$, Jordan M Joy ${ }^{3}$, Clayton L Cruthirds ${ }^{4}$, Christopher M Lockwood ${ }^{5}$, \\ John A Rathmacher ${ }^{6,7}$, Martin Purpura' and Jacob M Wilson ${ }^{3^{*}}$
}

\begin{abstract}
Introduction: Extracellular adenosine triphosphate (ATP) stimulates vasodilation by binding to endothelial ATP-selective P2Y2 receptors; a phenomenon, which is posited to be accelerated during exercise. Herein, we used a rat model to examine how different dosages of acute oral ATP administration affected the femoral blood flow response prior to, during, and after an exercise bout. In addition, we performed a single dose chronic administration pilot study in resistance trained athletes.

Methods: Animal study: Male Wistar rats were gavage-fed the body surface area, species adjusted human equivalent dose (HED) of either $100 \mathrm{mg}(n=4), 400 \mathrm{mg}(n=4), 1,000 \mathrm{mg}(n=5)$ or 1,600 mg $(n=5)$ of oral ATP as a disodium salt (Peak ATP®, TSI, Missoula, MT). Rats that were not gavage-fed were used as controls ( $C T L, n=5)$. Blood flow was monitored continuously: a) 60 min prior to, b) during and c) 90 min following an electrically-evoked leg-kicking exercise. Human Study: In a pilot study, 12 college-aged resistance-trained subjects were given $400 \mathrm{mg}$ of ATP (Peak ATP® , TSI, Missoula, MT) daily for 12 weeks, and prior to an acute arm exercise bout at weeks 1, 4, 8, and 12. Ultrasonography-determined volumetric blood flow and vessel dilation in the brachial artery was measured at rest, at rest 30 minutes after supplementation, and then at 0, 3, and 6 minutes after the exercise.

Results: Animal Study: Rats fed 1,000 mg HED demonstrated significantly greater recovery blood flow $(p<0.01)$ and total blood flow AUC values $(p<0.05)$ compared to CTL rats. Specifically, blood flow was elevated in rats fed 1,000 mg HED versus CTL rats at 20 to 90 min post exercise when examining 10-min blood flow intervals $(p<0.05)$. When examining within-group differences relative to baseline values, rats fed the 1,000 mg and 1,600 mg HED exhibited the most robust increases in blood flow during exercise and into the recovery period. Human study: At weeks 1, 8, and 12, ATP supplementation significantly increased blood flow, along with significant elevations in brachial dilation.
\end{abstract}

Conclusions: Oral ATP administration can increase post-exercise blood flow, and may be particularly effective during exercise recovery.

\section{Background}

Adenosine- $5^{\prime}$-triphosphate (ATP) is involved in all aspects of biosynthesis in cells and acts as the primary intracellular energy source. Extracellular ATP and its metabolites are involved in regulating a variety of biological processes including cardiac function, neurotransmission, liver glycogen metabolism, muscle contraction and blood flow [1].

\footnotetext{
* Correspondence: jwilson06x@gmail.com

${ }^{3}$ Department of Health Sciences and Human Performance, The University of Tampa, 318 N Boulevard, Tampa, FL 33606, USA

Full list of author information is available at the end of the article
}

Oral ATP administration has been shown to improve muscular function. Most episodes of lower back pain arise from structures in the lumbar spine, including the paravertebral musculature. ATP is linked to accelerating recovery in people with lower back pain by improving muscular cell function and increased blood flow [2]. Oral ATP administration has been shown to have an early acting effect in sub-acute low back pain and has been approved in France as an adjunct in the treatment of lower back pain [2].

Supplementation of $225 \mathrm{mg}$ per day of enteric-coated ATP supplementation for 15 days resulted in increased 
total bench press lifting volume as well as within-group repetitions to failure on set one of three with $70 \%$ of 1RM [3]. Moreover, 15 days of $400 \mathrm{mg}$ per day of ATP supplementation reduced muscle fatigue and enabled a higher force output during repeated high-intensity bouts of exercise [4]. More recently, 12 weeks of $400 \mathrm{mg}$ of oral ATP disodium salt supplementation in resistancetrained athletes utilizing a periodized resistance-training program (RT) resulted in significant increases in lean body mass, muscle thickness, total strength and vertical jump power [5]. ATP also reduced protein breakdown and limited the loss of strength and power during an overreaching cycle [5].

Three distinct mechanisms-of-action have been proposed for orally administered ATP's ergogenic benefits: 1) ATP can increase blood flow, resulting in improved oxygen and nutrient delivery to the muscle [5] 2) ATP may increase muscular excitability [6]; 3) ATP can trigger signaling cascades for metabolic adaptation related to neuromuscular activity (phosphorylation of ERK1/2) (see Figure 1) [7]. However, it is unlikely that oral ATP administration will directly increase intramuscular ATP stores.

Erythrocytes function as an oxygen sensor, contributing to the regulation of skeletal muscle blood flow and oxygen delivery, by releasing ATP in proportion to the number of unoccupied oxygen binding sites in the hemoglobin molecule. ATP release results in vasodilation and greater blood flow to the working musculature, thereby enhancing nutrient and oxygen delivery. Thus, during exercise under hypoxic conditions, ATP is released from the red blood cells via pannexin channels. ATP then binds to the purinergic receptors on the endothelial cells [5]. The endothelial cells then produce endothelium-derived hyperpolarizing factor, prostacyclin, and nitric oxide, all of which serve to relax the smooth muscle of the vasculature (see Figure 1) [5]. Infused ATP has been shown to increase blood flow by stimulating endothelial ATP-selective $\mathrm{P}_{2} \mathrm{Y}_{2}$ receptors and increasing muscle sympathetic vasoconstrictor activity [8]. The vasodilatory and sympatholytic effects of exogenous ATP are mediated via ATP itself rather than its dephosphorylated metabolites [9]. Chronic oral administration of ATP in rats increased portal vein ATP concentration and nucleoside uptake by erythrocytes, which resulted in an increase in ATP synthesis in the erythrocytes [10]. To our knowledge, however, no studies have delineated if oral ATP administration enhances the blood flow response to exercise.

This study used a rat model to examine how different dosages of acute oral ATP administration affected the femoral blood flow response prior to, during, and after an exercise bout. In addition, we performed a single dose chronic administration pilot study in resistance trained athletes.

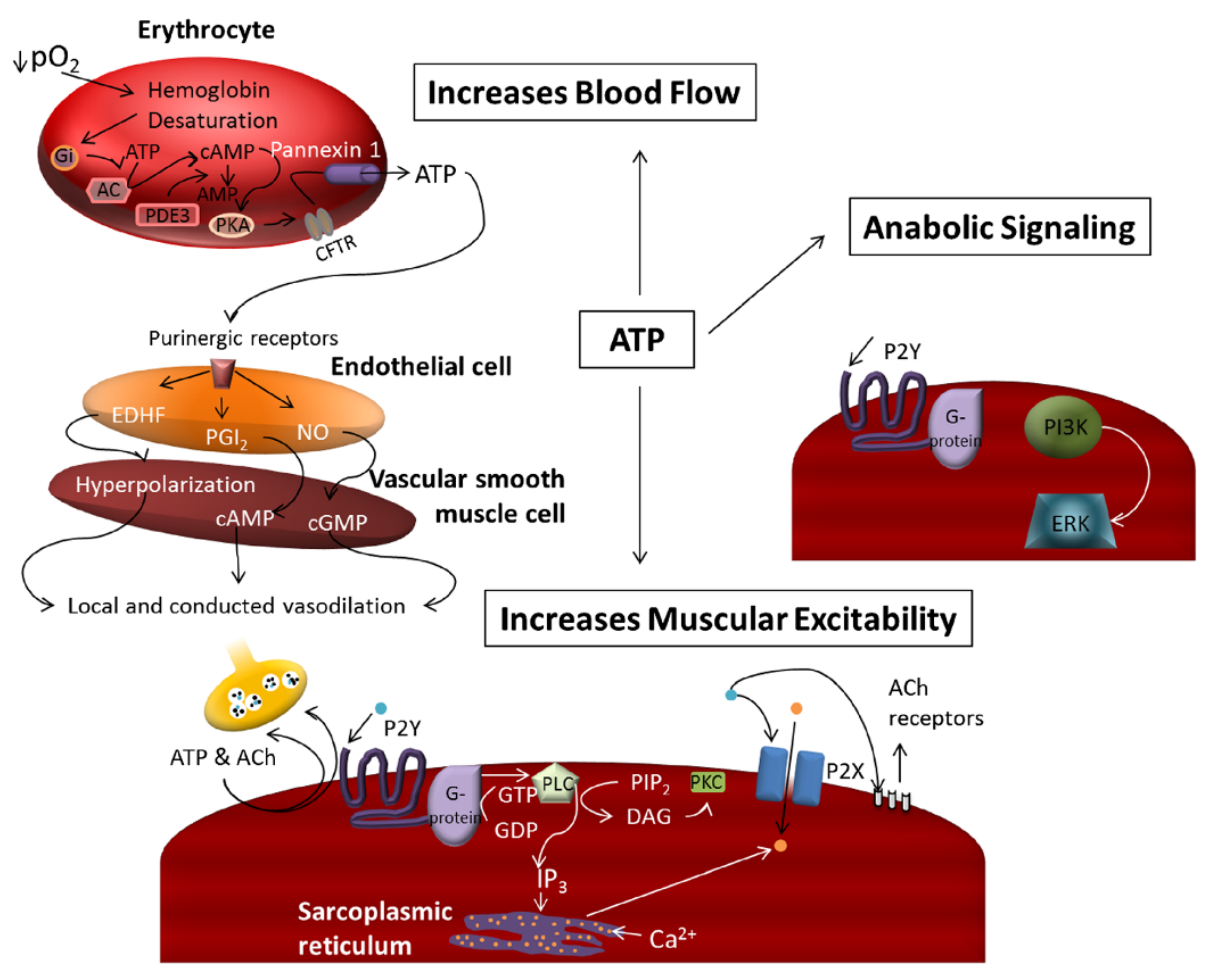

Figure 1 Proposed mechanism-of-action of oral ATP administration. 


\section{Methods}

\section{Animals and experimental protocol}

All animal work was conducted in the Department of Biomedical Sciences at the University of Missouri and was approved by the University of Missouri's Animal Care and Use Committee. Male Wistar rats were obtained from Charles River Laboratory weighing 250 g. Rats were allowed 7 days to acclimatize to new housing and were maintained on a 12/12-h light/dark cycle, with food (Harlan Laboratories, Tekland Global 14\% Rodent Maintenance diet) provided ad libitum until the experimental testing day. On the morning of testing, rats had food removed from homes cages at the beginning of the light cycle. Eight hours later, each rat was placed under isoflurane anesthesia and gavage-fed one of the following in $2 \mathrm{ml}$ of water: $3 \mathrm{mg}$ ATP (human equivalent dose of $100 \mathrm{mg}$ ), $\mathrm{n}=4 ; 12 \mathrm{mg}$ ATP (human equivalent dose of $400 \mathrm{mg}$ ), $\mathrm{n}=4 ; 31 \mathrm{mg}$ ATP (human equivalent dose of $1,000 \mathrm{mg}$ ), $\mathrm{n}=5 ; 49 \mathrm{mg}$ ATP (human equivalent dose of $1,600 \mathrm{mg}), \mathrm{n}=5$ or water only, $\mathrm{n}=5$ (CTL). All human equivalent doses administered were based upon body surface area conversion factors provided by Reagan-Shaw et al. [11].

Following feeding, a blood flow probe (Transonic Systems, Ithica, NY) was subsequently placed on the proximal portion of the right femoral artery and stimulation electrodes were placed in the right gastrocnemius muscle for an electrically-evoked plantarflexion exercise bout. Blood flow was then monitored continuously: a) $60 \mathrm{~min}$ prior to an electrically-evoked leg-kicking exercise (60 V, $100 \mathrm{pps}$, for $3 \mathrm{~min}$ for a total of 180 contractions), b) during the leg kicking exercise, and c) 90 min following exercise. This exercise bout was chosen per previous literature demonstrating that this protocol elicited an increase in femoral blood flow velocity in rats [12].

\section{Subjects and experimental protocol}

All human work was conducted in Department of Health Sciences and Human Performance at the University of Tampa and the protocol was approved by The University of Tampa Institutional Review Board. In a pilot study, 12 resistance-trained male participants (age $23.7 \pm 3.6$ years; height $179.0 \pm 1.0 \mathrm{~cm}$; weight $87.3 \pm 6.1 \mathrm{~kg}$ ) were given $400 \mathrm{mg}$ of ATP as a disodium salt (Peak ATP ${ }^{\oplus}$, TSI, Missoula, MT) daily 30 minutes before breakfast for 12 weeks. In addition at the beginning of the study and at weeks $1,4,8$, and 12 subjects consumed the $400 \mathrm{mg}$ of ATP 30 minutes prior to an acute elbow flexor bout (3 sets of 20 contractions at $50 \%$ of the subject's 1-RM). Measurements were taken at weeks $0,1,4,8$, and 12 . Ultrasonography-determined volumetric blood flow and vessel dilation in the brachial artery [13] was measured at rest before taking the supplement, at rest 30 minutes after supplementation, and then at 0,3 , and 6 minutes after the exercise . An ultrasound Doppler (LOGIQ e 2008, GE Healthcare, Wauwatosa, WI, USA) equipped with an annular phased array transducer probe (12-mm diameter), operating at an imaging frequency of $7.5 \mathrm{MHz}$ and variable Doppler frequencies of 4.0-6.0 MHz, was utilized to measure two-dimensional (2D) brachial arterial diameter and mean blood velocity at rest and following a one arm elbow flexor exercise bout. The depth range of the ultrasound beam was greater than the anatomic location of the brachial artery. Blood flow $\left(\mathrm{Q}=\right.$ vmean $\cdot \mathrm{A} \cdot 6 \times 10^{4}$, where vmean is mean blood velocity; $1 / \mathrm{min}$ ) was calculated from the amplitude (A) (signal intensity)-weighted, time- and spatial- averaged vmean $(\mathrm{m} / \mathrm{s})$, corrected for its angle of insonation, and multiplied by $\mathrm{A}\left(\mathrm{m}^{2}\right)$ of the brachial artery. The intraclass correlation coefficient (ICC) for the test-retest of blood flow and brachial arterial diameter ranged from 0.91 to 0.93 . The subjects were fully informed of any risks and discomforts associated with the experiments before giving their informed written consent to participate. All subjects worked with a registered dietician and were placed on a diet consisting of $25 \%$ fat, $25 \%$ protein, and $50 \%$ carbohydrates. Inclusion/exclusion criteria indicated that subjects had to have a minimum of 3 years of resistance training experience and could not be taking any nutritional supplements throughout the study. All subjects were told to maintain their normal training volume throughout the study.

\section{Statistics}

For the rat study, a two-way (treatment $\mathrm{x}$ time) mixed factorial ANOVA with LSD post hoc analysis was performed to determine if blood flow differed between treatments at each 10-min post-gavage interval. If a significant group, time, or group $\mathrm{x}$ time interaction existed the following statistical analyses were performed to further decompose the data: 1) individual independent samples t-tests were performed between treatments at each time point and significance was set at $\mathrm{p}<0.01$ in order to correct for an inflated type I error rate; 2) dependent t-tests were performed within treatments whereby each time point was compared to the baseline (-60 to $-50 \mathrm{~min}$ ) femoral artery blood flow values. For the rat study, mean femoral artery blood flow areas under the pre-exercise, exercise, post-exercise, and total blood flow curves (AUC) were also computed using SigmaPlot 12.0 which uses the trapezoidal rule algorithm for AUC calculations. Respective AUC values were compared between treatments using one-way ANOVAs with LSD post-hoc analyses where appropriate. All data were expressed as means \pm standard error values and significance was set at $\mathrm{p}<0.05$. For the human data we used a repeated measures analysis of variance using Statistica (StatSoft ${ }^{\oplus}$, Tulsa, OK, USA) to determine week, time, and week $\mathrm{X}$ time effects with an alpha 
level of 0.05. A tukey post-hoc for pairwise comparisons was run in the event of a significant F-test.

\section{Results}

\section{Animal data}

There were significant group $(\mathrm{p}<0.001)$ and time $(\mathrm{p}<0.001)$ effects, though no interaction effect $(\mathrm{p}>0.05)$. When examining within-group differences relative to baseline values, ATP supplementation, independently from the dose, did not increase blood flow pre-exercise. Exercise significantly increased blood flow in all groups at all time points during exercise compared to baseline values within each treatment $(\mathrm{p}<0.05) .3 \mathrm{mg}$ ATP had no effect on blood flow during the recovery period. $12 \mathrm{mg}$ ATP ( $<<0.001), 31 \mathrm{mg}$ ATP $(\mathrm{p}=0.003)$, and $49 \mathrm{mg}$ ATP $(\mathrm{p}<0.001)$ significantly increased blood flow 0 to $10 \mathrm{mi}-$ nutes post-exercise compared to baseline values within each treatment. In addition, $49 \mathrm{mg}$ ATP significantly increased blood flow 10 to 20, and 20 to 30 minutes post-exercise $(\mathrm{p}<0.05)$ compared to baseline values. Between-group comparisons at each time interval revealed that mean arterial blood flow was elevated in rats fed $31 \mathrm{mg}$ versus Ex/CTL rats at 30 to $90 \mathrm{~min}$ post exercise when examining 10-min blood flow intervals $(\mathrm{p}<0.01$ to $<0.001$; Figure 2 ).

Rats fed $31 \mathrm{mg}$ demonstrated significantly greater recovery blood flow $(\mathrm{p}=0.007)$ and total blood flow AUC values $(p=0.048)$ compared to CTL rats (Figure 3$)$.

\section{Human data}

At week 1 there was significant increase in blood flow at 0 min post exercise (Figure $4 ; p<0.01$ ) and tended to be increased at $3 \mathrm{~min}$ post exercise $(\mathrm{p}=0.07)$ in the ATP supplemented relative to the control week (week 0 ). This increase in brachial blood flow at week 1 was in conjunction with a significant elevation in brachial dilation at 0 min post exercise (Figure $5 ; \mathrm{p}<0.01$ ). After 8 weeks of ATP supplementation blood flow tended to be increased at 0 min post exercise $(\mathrm{p}=0.07)$ and was significantly increased at 3 min post exercise at 8 weeks and again at 12 weeks $(\mathrm{p}<0.01$ and $\mathrm{p}<0.05$, respectively) relative to the control week. This again was in concurrence with a significant increase in brachial dilation 30 min after ATP treatment at rest at week $8(\mathrm{p}<0.05)$ and 6 min post exercise at 12 weeks $(\mathrm{p}<0.01)$ and tended to be increased 0 post exercise at 8 and 12 weeks $(\mathrm{p}<0.10)$ relative to the control week.

\section{Discussion}

Wilson et al. recently suggested that oral ATP supplementation can significantly impact athletic performance, skeletal muscle hypertrophy and recovery; however, the study did not utilize methodologies to investigate the potential mechanism for the observed ergogenic effects [6]. One of the proposed mechanisms of action of oral ATP administration is an increase in blood flow, resulting in improved oxygen and nutrient delivery to the

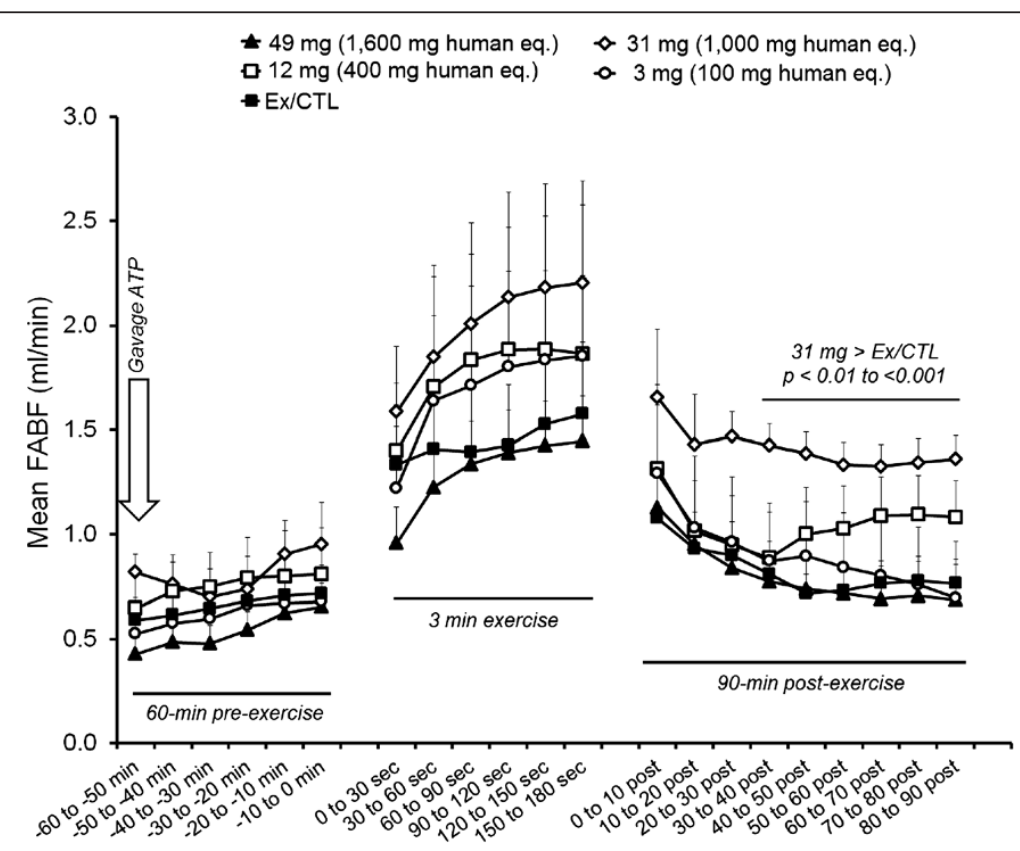

Figure 2 Mean femoral artery blood flow (FABF) values for $10 \mathrm{~min}$ intervals 60 to 0 minutes prior to exercise, during the 3-minute e-stim. exercise bout, and 0 to $\mathbf{9 0}$ min following exercise. Exercise increased blood flow within all groups compared to baseline values. Independent t-tests with correction for multiple comparisons revealed that $31 \mathrm{mg}$ of oral ATP prolonged femoral artery blood flow compared to Ex/CTL rats 30 to 90 min post-exercise $(p<0.01$ to $p<0.001)$. All data are presented as means \pm standard errors; $n=4-5$ animals per group. 


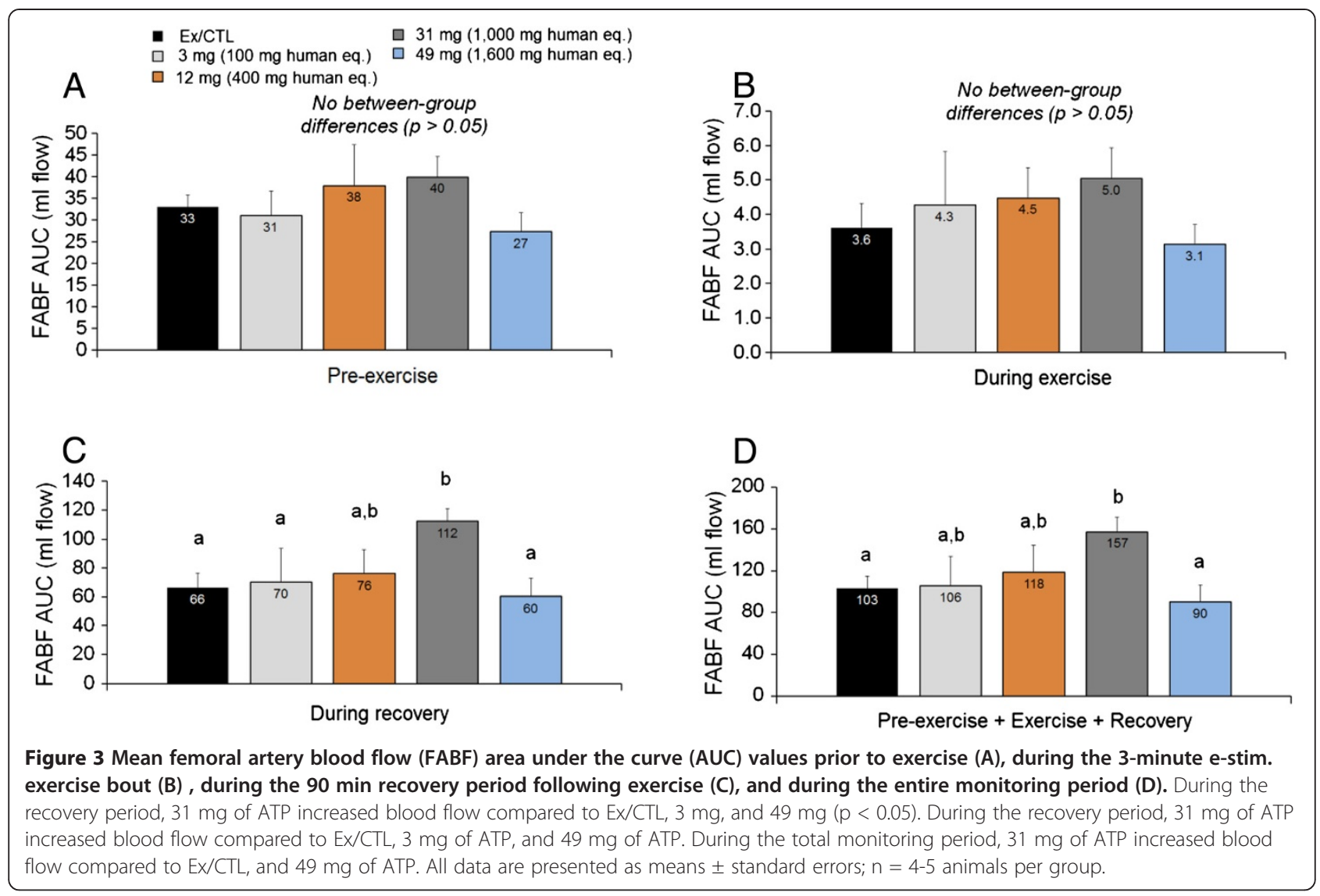

muscle. Enhanced blood flow to an exercising skeletal muscle is expected to improve removal of metabolic waste products such as lactate and urea. Following exercise nutrient delivery and cell swelling play a vital role in the skeletal muscle adaptation response. Improvements in blood flow conceivably would allow for greater delivery of nutrients for skeletal muscle repair following a muscle damaging bout of training resulting in increases in muscle hypertrophy previously seen with oral ATP administration. The main finding of this study was that

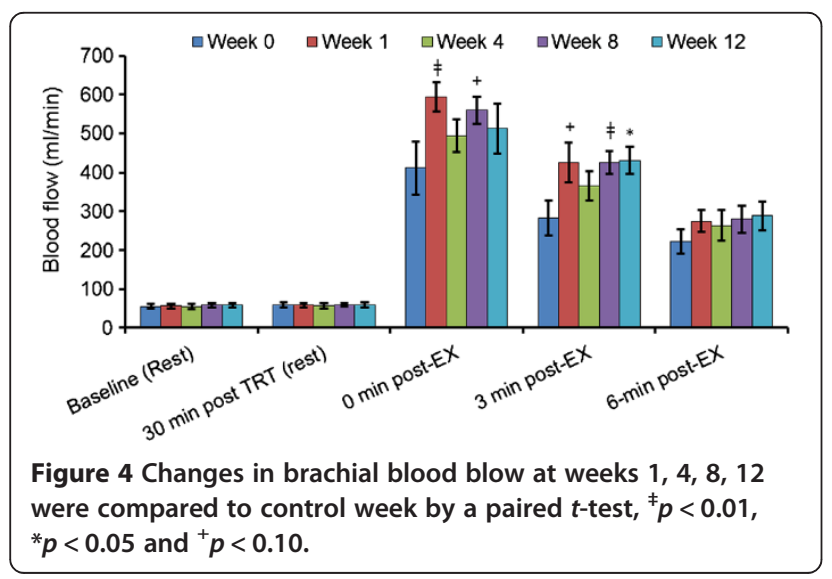

orally administered ATP as a disodium salt indeed increases blood flow in exercising animals and humans, most prominently during the recovery period from exercise. Significant improvements could be measured at a daily dose of $400 \mathrm{mg}$ ATP in as little as one week in the human study.

Though the exact mechanism of oral ATP absorption is currently not fully understood, animal studies have shown that the chronic oral administration of ATP resulted in measurable changes in muscle metabolism,

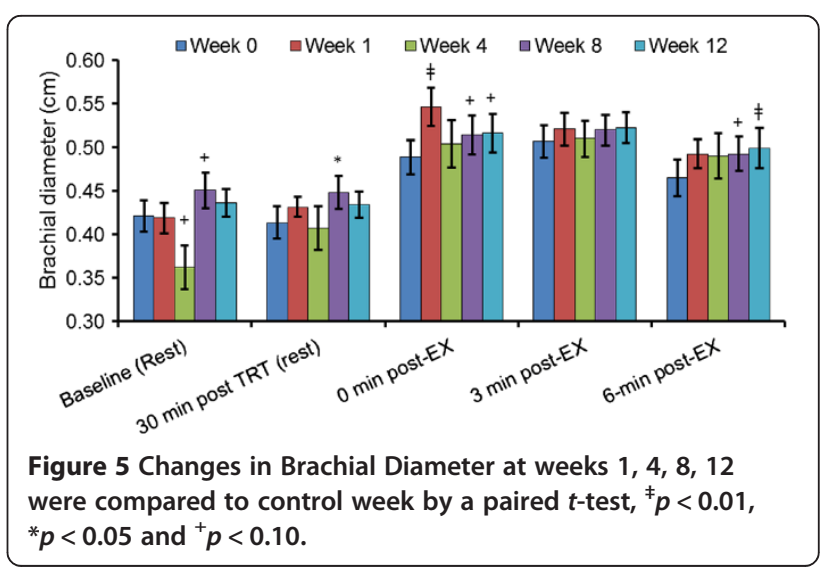


peripheral blood flow, and blood oxygenation $[10,14]$ and human studies have resulted in significant improvements in body composition and performance $[4,6]$. Studies on the oral availability of ATP showed that it is unlikely that oral ATP administration will directly increase intramuscular ATP stores as a single dose of orally administered ATP in humans did not increase ATP concentrations in blood [15]. The measurement of circulating free plasma ATP derived from oral ATP supplementation is very unlikely because exogenous free ATP is rapidly taken up by blood components or is rapidly metabolized. Kichenin et al. showed, in rats, that chronic oral administration of ATP increased portal vein ATP concentration and nucleoside uptake by erythrocytes, which resulted in an increase in ATP synthesis in the erythrocytes [10]. In other animal studies the administration of oral ATP resulted in a rapid degradation through ectonucleotidase triphosphatase diphosphohydrolase 1 (CD39), present on the luminal side of intestinal enterocytes, which dephosphorylate ATP via ADP to AMP, after which ecto-5' nucleotidase (CD73) degrades AMP to adenosine [16,17]. Following absorption of adenosine and inorganic phosphate in the small intestine and the portal circulation these moieties are then incorporated into liver ATP pools, leading to expansions of these pools. Therefore, the systemic and oral administrations of ATP result in the expansions of liver, blood (red blood cells) and blood plasma (extracellular) pools of ATP which were shown for the first time by Rapaport et al. [18,19].

Blood flow during exercise is indicative of nutrient (amino acids, glucose, etc.) and oxygen delivery rate. As such, increased blood flow will indicate greater nutrient availability for the working musculature, and, in theory, the muscle should have the capacity to recover more quickly between sets, maintain performance longer, and repair microtrauma more efficiently between training sessions. Wilson et al. [6] hypothesized that the observed increases in lean body mass, markers of athletic performance, and resistance to an overreaching status with chronic ATP supplementation were due to enhanced blood flow leading to enhanced recovery, although this remained to be directly examined until the current study. However, despite increased blood flow during ATP infusion, oxygen consumption does not increase [20]. Considering these two studies, it is possible that ATP is more efficacious for anaerobic versus aerobic based exercise. However, ATP's efficacy in an endurance model remains to be investigated. Likewise, the exact mechanism whereby ATP increases post-exercise blood flow also remains to be determined, although others have hypothesized that this may be due to: a) ATP degradation products being taken up by erythrocytes and resynthesized into ATP; b) vasodilation of ATP degradation (i.e., adenosine) products; and/or c) adenosine-stimulated nitric oxide and prostacyclin synthesis and downstream signaling [4].

L-citrulline or L-arginine are amino acid precursors to nitric oxide and have been marketed as potential ergogenic aids based on their ability to increase blood flow to the exercising muscle. However, the daily dose needed to increase blood flow is high (6-24 g) and the ergogenic response may depend on the training status and health of the subjects [21]. Whereas some studies involving untrained or moderately healthy subjects showed that nitric oxide donors could improve tolerance to aerobic and anaerobic exercise, no significant improvements were measured in healthy [22] or highly-trained subjects [21,23]. In contrast, oral ATP increases blood flow at mg doses and has been shown to increase lean body mass, strength and power in highly trained individuals [6]. Therefore, oral ATP supplementation is an apparently efficacious method if the intent is increasing post-exercise blood flow and nutrient delivery.

Limitations of this study include the lack of control group and a rigorous control of other potentially confounding variables such as potential differences in exercise habits or baseline dietary habits and dietary supplement use in the human data. We propose future research to assess the effects of oral ATP administration on blood flow in a placebo-controlled crossover or parallel design.

\section{Conclusion}

Oral ATP administration can increase blood flow, and this effect is particularly prominent following exercise. Increased blood flow due to ATP supplementation may be the mechanism responsible for ergogenic effects following chronic ATP supplementation as previously reported in the scientific literature. However, the exact mechanism whereby ATP increases blood flow during post-exercise recovery periods remains unknown and future investigation in this area is warranted.

\section{Competing interests}

JMW, JMJ, RPL, MDR, and CLC declare no competing interests. JR is employed by Metabolic Technologies, Inc. which engages in business trade with TSI (USA), Inc. RJ and MP are, and CML was a consultant of TSI, Inc

\section{Authors' contribution}

The manuscript was written through contributions of all authors. All authors have given approval to the final version of the manuscript.

\section{Acknowledgements}

We are grateful for the support from TSI, Missoula, MT, for funding this study.

\section{Author details}

${ }^{1}$ Increnovo LLC, 2138 E Lafayette PI, Milwaukee, WI 53202, USA. ${ }^{2}$ School of Kinesiology, Molecular and Applied Sciences Laboratory, Auburn University, Auburn, AL 36849, USA. ${ }^{3}$ Department of Health Sciences and Human Performance, The University of Tampa, 318 N Boulevard, Tampa, FL 33606 , USA. ${ }^{4}$ Department of Biomedical Sciences, College of Veterinary Medicine, University of Missouri-Columbia, 1600 Rollins, Columbia, MO 65211, USA. ${ }^{5} 4$ Life Research, 9850 S 300 W, Sandy, UT 84070, USA. ${ }^{6}$ Metabolic Technologies Inc., 2711 S Loop Dr, Suite 4400, Ames, IA 50010, USA. ${ }^{7}$ Department of Animal Sciences, lowa State University, Ames, IA, 50011, USA. 
Received: 26 February 2014 Accepted: 2 June 2014

Published: 13 June 2014

\section{References}

1. Agteresch HJ, Dagnelie PC, van den Berg JW, Wilson JH: Adenosine triphosphate: established and potential clinical applications. Drugs 1999, 58(2):211-232.

2. Bannwarth B, Allaert F-A, Avouac B, Rossignol M, Rozenberg S, Valat J-P: A randomized, double-blind, placebo controlled study of oral adenosine Triphosphate in subacute low back pain. J Rheumatol 2005, 32:1114-1117.

3. Jordan AN, Jurca R, Abraham EH, Salikhova A, Mann JK, Morss GM, Church TS, Lucia A, Earnest CP: Effects of oral ATP supplementation on anaerobic power and muscular strength. Med Sci Sports Exerc 2004, 36(6):983-990.

4. Rathmacher JA, Fuller JC Jr, Baier SM, Abumrad NN, Angus HF, Sharp RL: Adenosine-5'-triphosphate (ATP) supplementation improves low peak muscle torque and torque fatigue during repeated high intensity exercise sets. J Int Soc Sports Nutr 2012, 9(1):48.

5. Sprague RE, Bowles EA, Achilleus D, Ellsworth ML: Erythrocyte as controllers of perfusion distribution in the microvasculature skeletal muscle. Acta Physiol 2011, 202:285-292.

6. Wilson JM, Joy JM, Lowery RP, Roberts MD, Lockwood CM, Manninen AH, Fuller JC Jr, De Souza EO, Baier SM, Wilson SMC, Rathmacher JA: Effects of oral adenosine-5'-triphosphate (ATP) supplementation on athletic performance, skeletal muscle hypertrophy and recovery in resistance-trained men. Nutr Metab (Lond) 2013, 10:57.

7. May C, Weigl L, Karel A, Hohenegger M: Extracellular ATP activates ERK1/ ERK2 via a metabotropic P2Y1 receptor in a Ca2+ independent manner in differentiated human skeletal muscle cells. Biochem Pharmacol 2006, 71(10):1497-1509.

8. Rosenmeier JB, Hansen J, Gonźalez-Alonso J: Circulating ATP-induced vasodilatation overrides sympathetic vasoconstrictor activity in human skeletal muscle. J Physiol 2004, 558:351-365.

9. Rosenmeier JB, Yegutkin GG, Gonźalez-Alonso J: Activation of ATP/UTP-selective receptors increases blood flow and blunts sympathetic vasoconstriction in human skeletal muscle. J Physiol 2008, 586:4993-5002.

10. Kichenin K, Seman M: Chronic oral administration of ATP modulates nucleoside transport and purine metabolism in rats. J Pharmacol Exp Ther 2000, 294(1):126-133.

11. Reagan-Shaw $\mathrm{S}$, Nihal $M, A$ hmad N: Dose translation from animal to human studies revisited. FASEB J 2008, 22(3):659-661.

12. Mohr T, Akers TK, Wessman HC: Effect of high voltage stimulation on blood flow in the rat hind limb. Phys Ther 1987, 67(4):526-533.

13. Corretti MC, Anderson TJ, Benjamin EJ, Celermajer D, Charbonneau F, Creager MA, Deanfield J, Drexler H, Gerhard-Herman M, Herrington D, Vallance P, Vita J, Vogel R: Guidelines for the Ultrasound Assessment of Endothelial-Dependent Flow-Mediated Vasodilation of the Brachial Artery. J Am Coll Cardiol 2002, 39:257-265.

14. Kichenin K, Decollogne S, Angignard J, Seman M: Cardiovascular and pulmonary response to oral administration of ATP in rabbits. $J$ Appl Physiol 2000, 88:1962-1968.

15. Arts IC, Coolen EJ, Bours MJ, Huyghebaert N, Stuart MA, Bast A, Dagnelie PC: Adenosine 5 '-triphosphate (ATP) supplements are not orally bioavailable: a randomized, placebo-controlled cross-over trial in healthy humans. $J$ Int Soc Sports Nutr 2012, 9(1):16.

16. Yegutkin GG: Nucleotide- and nucleoside-converting ectoenzymes: important modulators of purinergic signalling cascade. Biochim Biophys Acta 2008, 1783:673-694

17. Strohmeier GR, Lencer WI, Patapoff TW, Thompson LF, Carlson SL, Moe SJ, Carnes DK, Mrsny RJ, Madara JL: Surface expression, polarization, and functional significance of CD73 in human intestinal epithelia. J Clin Invest 1997, 99:2588-2601.

18. Rapaport E, Fontaine J: Anticancer activities of adenine nucleotides in mice are mediated through expansion of erythrocyte ATP pools. Proc Natl Acad Sci U S A 1989, 86(5):1662-1666.

19. Rapaport E, Fontaine J: Generation of extracellular ATP in blood and its mediated inhibition of host weight loss in tumor-bearing mice. Biochem Pharmacol 1989, 38(23):4261-4266.

20. Calbet JA, Lundby C, Sander M, Robach P, Saltin B, Boushel R: Effects of ATP-induced leg vasodilation on VO2 peak and leg $\mathrm{O} 2$ extraction during maximal exercise in humans. Am J Physiol Regul Integr Comp Physiol 2006, 291(2):R447-R453.
21. Sureda A, Pons A: Arginine and citrulline supplementation in sports and exercise: ergogenic nutrients? Med Sport Sci 2012, 59:18-28.

22. Tang JE, Lysecki PJ, Manolakos JJ, MacDonald MJ, Tarnopolsky MA, Phillips SM: Bolus arginine supplementation affects neither muscle blood flow nor muscle protein synthesis in young men at rest or after resistance exercise. J Nutr 2011, 141(2):195-200.

23. Alvares TS, Conte CA, Paschoalin VM, Silva JT, Meirelles Cde M, Bhambhani YN, Gomes PS: Acute l-arginine supplementation increases muscle blood volume but not strength performance. Appl Physiol Nutr Metab 2012, $37(1): 115-126$

doi:10.1186/1550-2783-11-28

Cite this article as: Jäger et al:: Oral adenosine-5'-triphosphate (ATP) administration increases blood flow following exercise in animals and humans. Journal of the International Society of Sports Nutrition 2014 11:28.

\section{Submit your next manuscript to BioMed Central and take full advantage of:}

- Convenient online submission

- Thorough peer review

- No space constraints or color figure charges

- Immediate publication on acceptance

- Inclusion in PubMed, CAS, Scopus and Google Scholar

- Research which is freely available for redistribution

Submit your manuscript at www.biomedcentral.com/submit
C) Biomed Central 